\title{
Revue de livre
}

\section{Anne E. Calvès, Fatou Binetou Dial et Richard Marcoux (dir.), Nouvelles dynamiques familiales en Afrique, Québec: Presses de l'Université du Québec, 2018, 419 pages.}

Véronique Senécal-Lirette

Chercheure indépendante

n raison des changements sociaux et économiques qui se sont déroulés au
tournant du XIX ${ }^{\mathrm{e}}$ siècle, de nombreuses restructurations sociales et démographiques se sont produites en Afrique subsaharienne (Mondain, Delaunay et Adjamagbo 2009). La crise économique des années I980-I990, la mondialisation, les politiques d'austérité, la plus grande scolarisation des femmes africaines et une remise en question des normes traditionnelles ambiantes qui s'en sont suivies ont contribué à amener ces changements (Locoh et Puech 2008). L'ouvrage Nouvelles dynamiques familiales en Afrique fait suite aux contributions présentées à l'atelier-colloque sur les dynamiques familiales en Afrique subsaharienne qui a eu lieu à Dakar, du I8 au 20 décembre 20I6, organisé par le Groupe interuniversitaire d'études et de recherches sur les sociétés africaines (GIERSA), avec l'Institut fondamental d'Afrique noire (IFAN) de l'Université Cheikh Anta Diop. L'objectif de l'ouvrage est de dresser un portrait des transformations familiales qui s'opèrent actuellement dans les sociétés africaines subsahariennes, tout en se penchant sur les enjeux que ces changements amènent.

Le collectif est composé de 17 chapitres regroupant 35 contributeurs et s'articule autour de quatre axes: structures des ménages et configurations familiales; emploi des femmes et conciliation travail-famille; parentalité, socialisation des enfants et rapports intergénérationnels; et enfin, politiques, programmes et droit de la famille. Ceux-ci présentent des réalités dans quelques pays africains, notamment le Sénégal, le Mali, le Burkina Faso, le Congo, le Bénin, le Togo et le Cameroun, suivant différents champs d'expertise des sciences humaines et sociales. Le premier chapitre, rédigé par Philippe Antoine, porte sur la polygamie urbaine et rurale au Sénégal. Celui-ci montre certaines failles que peuvent 
avoir les enquêtes de sondage pour rapporter de façon efficace un tel sujet. Il souligne l'importance de ne pas uniquement se baser sur les approches qui s'appuient sur les liens de parenté des personnes qui résident avec le chef de ménage. Le deuxième chapitre (Cudeville, Guénard et Robillard) aborde la polygamie, la corésidence des époux et l'offre de travail des femmes au Sénégal. Il démontre que le nombre d'heures travaillées et la charge domestique des femmes varient en fonction du statut matrimonial et du mode de résidence. Le troisième chapitre (Delaunay et ses collègues) porte, quant à lui, sur la monoparentalité au Bénin, au Burkina Faso et au Togo. Le concept de monoparentalité hébergée y est proposé, lequel peut être relié à un arrangement résidentiel ou à l'instabilité matrimoniale créée, par exemple, suite à une séparation, un veuvage ou une naissance prémaritale. Le quatrième chapitre (Moguérou, Wayack-Pambè et Awissi) aborde la situation des femmes célibataires et cheffes de ménages à Dakar et à Ouagadougou et la manière dont elles sont perçues.

Le cinquième chapitre (Jacquemin et ses collègues) porte sur la conciliation travail-famille des femmes à Dakar et démontre que la féminisation du marché du travail n'engendre ni une réorganisation des rôles de chacun au sein du ménage, ni un partage des tâches domestiques. Le chapitre suivant, rédigé par Bilampoa G. Thiombiano, aborde le travail et la famille en milieu urbain. L'auteure y fait ressortir la précarité des conditions de travail chez les hommes et les femmes, ces dernières se retrouvant plus fragilisées alors qu'elles occupent régulièrement des emplois dans le secteur informel tout en s'occupant de la charge de travail domestique et du soin des enfants. Sous le poids des traditions, cette réalité semble perpétuée tant par les hommes que par les femmes ellesmêmes. Le septième chapitre, écrit par Ayemi Lawani, porte sur la conciliation travail-famille, à Cotonou et à Lomé, chez des femmes travaillant au sein d'ONG de développement. Le chapitre qui suit, écrit par Gning et ses collègues, présente les trajectoires familiales et les dynamiques des réseaux sociaux d'entrepreneures à Saint-Louis du Sénégal. Deux typologies de femmes entrepreneures y sont présentées, soit les héritières et les résilientes. Dans certains cas, l'appui et l'apport de figures masculines du réseau familial et des leaders locaux sont essentiels. Les enfants sont aussi amenés à augmenter la visibilité de leurs mères sur internet et à négocier et commercialiser ce qu'elles produisent.

Le neuvième chapitre, écrit par Doris Bonnet, explore le sujet de la construction d'une famille dans le contexte du recours à la reproduction médicale à Douala, au Cameroun, et de la remise en question de la pratique de confier un enfant. Le recours à la reproduction médicale apparaît ainsi comme une 
possibilité d'avoir un enfant «à elles» alors que le don d'ovocytes est admis, mais que le don de sperme provoque beaucoup plus de réticences. Le dixième chapitre, rédigé par Anne E. Calvès et Afiwa N'Bouké, montre que le mariage n'est plus la voie exclusive vers la fondation d'une famille. Le nombre grandissant d'unions libres s'expliquerait par une remise en cause des normes traditionnelles et par l'autonomisation financière des femmes. Le onzième chapitre est écrit par Fatou Binetou Dial. Celui-ci met en lumière le peu de structures de prise en charge des aînés au Sénégal, une situation amenée à changer avec l'allongement de l'espérance de vie et la baisse de fécondité. Ainsi, les jeunes se voient bien souvent obligés de prendre soin de leurs parents, ce qui peut représenter un poids social et financier considérable. Le douzième chapitre (Sawadogo, Bazié et Guiella) porte sur la socialisation des filles et des garçons et sur les normes de genres transmises par les parents à Ouagadougou. Les auteurs démontrent que les normes et les stéréotypes de genre perdurent. Il est toutefois encourageant de constater certains changements à cet égard, alors que les parents constatent les nouvelles réalités sociales. Le treizième chapitre, par Moussa Bougma, s'interroge sur le fait que les enfants issus de ménages dirigés par une femme aient de meilleures chances de réussite scolaire que ceux provenant de ménages dirigés par un homme. Cette constatation s'explique par le fait que les mères sont plus sensibilisées à l'importance de la poursuite d'études et de la réussite scolaire chez les jeunes filles.

Le quatorzième chapitre, d'Ousmane Koné, examine les pressions exercées par les organisations islamiques maliennes, à l'aide de canaux de communications tels que les radios religieuses privées et les prêches, pour la modification du Code des personnes et de la famille, jugé influencé par des normes occidentales trop en faveur des femmes. Ces organisations auront d'ailleurs obtenu sa relecture qui mènera à une version adaptée en 20II. Le quinzième chapitre, de Marième N'Diaye, aborde la méconnaissance qu'ont les femmes sénégalaises de leurs droits. Si les textes de loi sont davantage progressistes quant aux droits des femmes, en pratique, au sein des tribunaux, il y a toujours un conservatisme sur le plan des normes de genre. L'avant-dernier chapitre, de Marie-Ève Paré, observe que la polygynie chez les Mossis est toujours bien présente dans les zones rurales, mais tend à diminuer dans les régions urbaines en raison des conjonctures économiques. L'étude souligne le faible taux d'enregistrement des mariages civils, alors que la législation cherchait à mieux protéger et faire valoir les droits des femmes. Le dernier chapitre (Pungi, White et Makobo) met en relief le fossé entre les valeurs familiales externes et les normes locales à 
travers l'analyse de feuilletons radiophoniques élaborés par l'ONG américaine Population Media Center diffusés au Congo. Ces tribunes, qui abordent certains sujets tels que la fécondité et la planification des naissances, n'ont pas atteint les objectifs visés, puisqu'elles outrageaient les mœurs locales.

En somme, l'ouvrage témoigne d'un certain détachement objectif: on y rapporte des faits sans y apposer de jugements de valeur, sans victimisation à outrance des femmes africaines ni diabolisation de l'Occident. Il est rafraîchissant de voir aussi que plusieurs chapitres sont écrits par des Africaines et des Africains, ce qui permet une réappropriation du savoir. S'il n'y avait qu'une seule critique à faire, ce serait certainement envers le chapitre de Fatou Binetou Dial où l'essentialisation des acteurs est plutôt dérangeante, et la polarisation entre les sociétés occidentales et africaines, lourde et réductrice.

Enfin, il est évident que le livre traite principalement des répercussions de ces changements sociétaux chez les femmes. Or, il aurait été intéressant de laisser davantage de place au vécu des hommes et de consacrer aussi quelques chapitres à la façon dont ces nouvelles dynamiques familiales influencent leur quotidien. Il s'agit toutefois, dans l'ensemble, d'un ouvrage très pertinent et qui présente des écrits sur un large éventail de thèmes touchant aux nouvelles dynamiques familiales en Afrique.

\section{Références}

Locoh, Thérèse et Isabelle Puech, 2008. «Fatou Sow. Les défis d'une féministe en Afrique». Travail, genre et sociétés 20 (2) : 5-22. DOI: 10.3917/tgs.000.0004

Mondain, Nathalie, Valérie Delaunay et Agnès Adjamagbo, 2009. «Maternité et mariage en milieu rural sénégalais: Quel avenir pour les mères célibataires?». In Catherine Gourbain (dir.), Santé de la reproduction au Nord et au Sud: De la connaissance à l'action, p. III-I30. Louvain-la-Neuve, Université catholique de Louvain. 